\title{
Terrestrial aurora: astrophysical laboratory for anomalous abundances in stellar systems
}

\author{
I. Roth \\ Space Sciences, UC Berkeley CA 94720, USA \\ Correspondence to: I. Roth (ilan@ssl.berkeley.edu) \\ Received: 15 March 2013 - Revised: 31 August 2013 - Accepted: 16 September 2013 - Published: 11 February 2014
}

\begin{abstract}
The unique magnetic structure of the terrestrial aurora as a conduit of information between the ionosphere and magnetosphere can be utilized as a laboratory for physical processes at similar magnetic configurations and applied to various evolutionary phases of the solar (stellar) system. The most spectacular heliospheric abundance enhancement involves the ${ }^{3} \mathrm{He}$ isotope and selective heavy elements in impulsive solar flares. In situ observations of electromagnetic waves on active aurora are extrapolated to flaring corona in an analysis of solar acceleration processes of ${ }^{3} \mathrm{He}$, the only element that may resonate strongly with the waves, as well as heavy ions with specific charge-to-mass ratios, which may resonate weaker via their higher gyroharmonics. These results are applied to two observed anomalous astrophysical abundances: (1) enhanced abundance of ${ }^{3} \mathrm{He}$ and possibly ${ }^{13} \mathrm{C}$ in the late stellar evolutionary stages of planetary nebulae; and (2) enhanced abundance of the observed fossil element ${ }^{26} \mathrm{Mg}$ in meteorites as a decay product of radioactive ${ }^{26} \mathrm{Al}$ isotope due to interaction with the flare-energized ${ }^{3} \mathrm{He}$ in the early solar system.
\end{abstract}

Keywords. Solar physics, astrophysics, and astronomy (energetic particles)

\section{Introduction}

Terrestrial aurora has been observed over millennia as a bright glow at various wavelengths (colors) of spectacular shapes in the night sky, usually in the polar region. The aurora forms a unique feature in the naturally occurring magnetized configurations, linking and communicating between two distinctly different plasmas: ionosphere and magnetosphere. Terrestrial ionosphere is a part of the upper atmosphere, consisting of dense $(\sim 1000 / \mathrm{cc})$ plasma of charged ions (mainly $\mathrm{H}^{+}, \mathrm{O}^{+}$and $\mathrm{N}^{+}$) and electrons, formed mainly due to the solar ultraviolet radiation, with a small concentration of neutral molecules. The more dilute plasmas in the magnetospheres of the Earth, as well as at Mercury, Jupiter, Saturn, Uranus, Neptune, Jupiter's moon Ganymede and probably at the extra-solar planets fill regions in space whose shape is determined by the internal (planetary) magnetic field, the solar (stellar) wind and the interplanetary magnetic field. Magnetic connection between these regions allows the intense fluctuations in the solar wind, originating mostly at the Sun, or perturbations due to reconfiguration of the magnetospheric tail, to be transmitted along auroral field lines, resulting in the observed vivid illuminations, where down-flowing accelerated electron beams of $1-20 \mathrm{keV}$ impinge on atmospheric atoms/molecules at a low altitude of $\sim 80-100 \mathrm{~km}$ at Earth. The power of the terrestrial aurora is assessed at $1000 \mathrm{MW}$, with an output for Jupiter 1000 times larger, while Neptune's power is only 50 MW. Similarly, during electromagnetically active times, the excited waves heat the ionospheric ions, which flow up along the auroral lines, resulting in the observed terrestrial enrichment of magnetospheric ring current at energies of $20-50 \mathrm{keV}$ by the singly charged ionospheric ions, differentiating them from the multiply charged solar ions. Auroral electron beams collide with atmospheric gases, exciting their bound electrons to higher atomic levels, with subsequent emissions in the visible fluorescent range; intermittent emissions at the infrared, ultraviolet and in X-ray frequencies are recorded due to various molecular, atomic and nuclear excitations. The visible light is dominated by emissions from atomic oxygen in a greenish glow at a wavelength of $557.7 \mathrm{~nm}$ and dark-red glow at $630.0 \mathrm{~nm}$ (forbidden/delayed electronic transitions), and from atomic and molecular nitrogen (blue and purple, respectively). The timescales and the well-resolved locations 


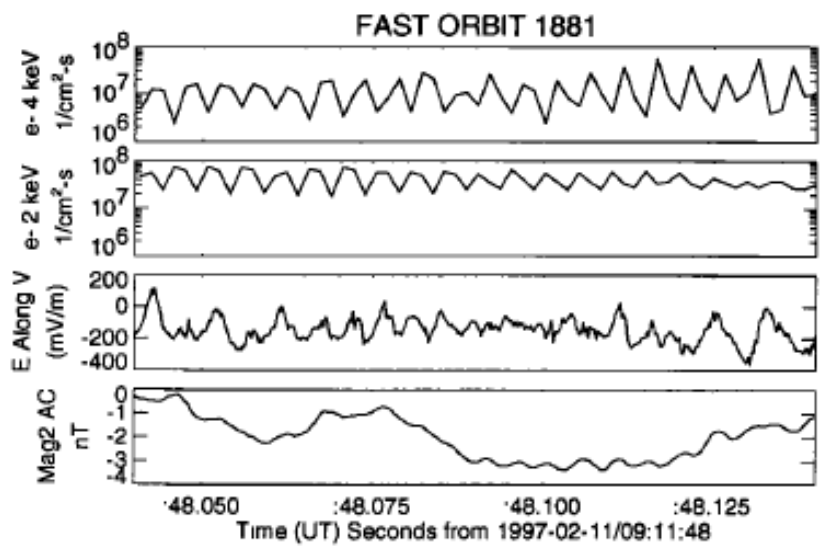

Fig. 1. Direct FAST satellite observation at the crossing of an auroral arc, measuring energetic electron fluxes and components of electric and magnetic fields (McFadden et al., 1998).

of the emissions reveal details about the energization processes.

Auroral magnetic configuration creates a unique laboratory for magnetized plasma processes, which may be applicable to other extraterrestrial environments, as well as to processes that take place during various evolutionary phases of the solar (stellar) system. Here we focus on a small subset of in situ auroral observations that may help in understanding the anomalous abundances of elements and isotopes at the solar, astrophysical and meteoric sites, with applications to the inception and to the final life stages of stellar systems.

\section{Auroral observations}

Auroral dynamic region of magnetized plasma with several ion species becomes naturally a source of rich plasma activity. Various satellites performed global imaging of the whole polar oval (e.g., Polar UV, IMAGE), while others (e.g., FAST) crossing auroral field lines offered good resolution of waves and particles. Particular observations include the fast temporal electron and wave oscillations, as shown on Fig. 1, at a frequency of $120 \mathrm{~Hz}$, below the hydrogen $\mathrm{H}^{+}$gyrofrequency at $\sim 208$, indicating that ions with gyrofrequencies around this frequency could be affected resonantly by these waves. Such ions are not found naturally in the geophysical environment, but they can be observed at solar or stellar coronae. If a wave-particle process similar to the terrestrial aurora operates at the stellar corona, these ions can be substantially heated. Additional selected charge states of heavier elements with a specific charge-to-mass ratio may interact resonantly with these waves through a multiple gyroharmonic interaction.

\section{Abundance anomalies in solar ions}

Observations of anomalous abundances of rare, energetic solar elements, or isotopes with unusual charge states present a challenge with astrophysical implications. Ions of solar origin with energies of 1.0-10.0 MeV/nucleon and beyond, many orders of magnitude above the solar wind energy, are observed intermittently in the interplanetary space. Their abundances and isotopic states are often distinctly different from the coronal or solar wind values, which characterize the galactic values. While most intense ion acceleration occurs in "gradual" events that span days and are correlated with the propagating interplanetary coronal mass ejection shocks, the most spectacular heliospheric enhancements with anomalous abundances involve "impulsive" solar flares with a duration of minutes to hours, being correlated with intense electron fluxes of 10-20 keV accompanying coronal release processes, deduced from Bremsstrahlung radiation. The main enhancements involve ${ }^{3} \mathrm{He}$, heavy elements with high charge states: $\mathrm{Fe}, \mathrm{Mg}, \mathrm{Ne}$ and $\mathrm{Si}$, higher mass isotopes (like ${ }^{22} \mathrm{Ne}$ ), and a subset of ultra-heavy $\mathrm{Xe}$ and $\mathrm{Kr}$ ions.

Primordial nucleo-synthesis and galactic evolution confines the coronal ratio of the He isotopes ${ }^{3} \mathrm{He} /{ }^{4} \mathrm{He}$ to several times $10^{-4}$; this ratio is enhanced in impulsive flares by a factor of $10^{3}-10^{5}$ (Hsieh and Simpson, 1970; Mason et al., 2000), while heavier ions (mainly Fe with higher charge state of 18-22 vs. 10-14 in solar wind) are enhanced by a factor up to 10 with respect to O (Mason et al., 1986; Reames et al., 1994). ACE satellite also observed impulsive events with ${ }^{3} \mathrm{He}$ and $\mathrm{Fe}$ of similar energy distributions, which differ from other ion spectra, indicating a commonality in their acceleration mechanisms. Some velocity spectrograms show that ${ }^{3} \mathrm{He}$ and $\mathrm{Fe}$ arrive along the same energy-time curves, confirming for these events a simultaneous injection at the Sun (Mason et al., 2000). A study of specific impulsive ${ }^{3} \mathrm{He}$-rich events concluded that ${ }^{3} \mathrm{He} /{ }^{4} \mathrm{He}$ and $\mathrm{Fe} / \mathrm{O}$ enhancements are clearly correlated (Ho et al., 2000).

There exists an interesting analogy between physical processes on active auroral and flaring coronal field lines (Temerin and Roth, 1992; Roth and Temerin, 1997). Both environments have very low $\beta$ (thermal to magnetic pressure ratio) plasmas, are dominated by two majority ion species $\left(\mathrm{H}^{+}\right.$and $\mathrm{O}^{+}$at Earth, $\mathrm{H}^{+}$and $\mathrm{He}^{++}$at the Sun), and large electron fluxes flow along the active respective configurations. In the corona these fluxes are deduced from X-ray emissions, while in the aurora they are measured in situ. Auroral observations indicate that oblique electromagnetic ion cyclotron waves with a relatively narrow spectrum are associated with the accelerated electrons that form the discrete aurora (Temerin and Lysak, 1984). Fisk (1978) invoked a one-stage resonant acceleration process on coronal field lines with electrostatic cyclotron waves, requiring unrealistically high ${ }^{4} \mathrm{He}$ density. Additional models of one-stage coronal acceleration include the stochastic acceleration of $\mathrm{Fe}$ by shear Alfven waves (Miller and Vinas, 1993), stochastic 


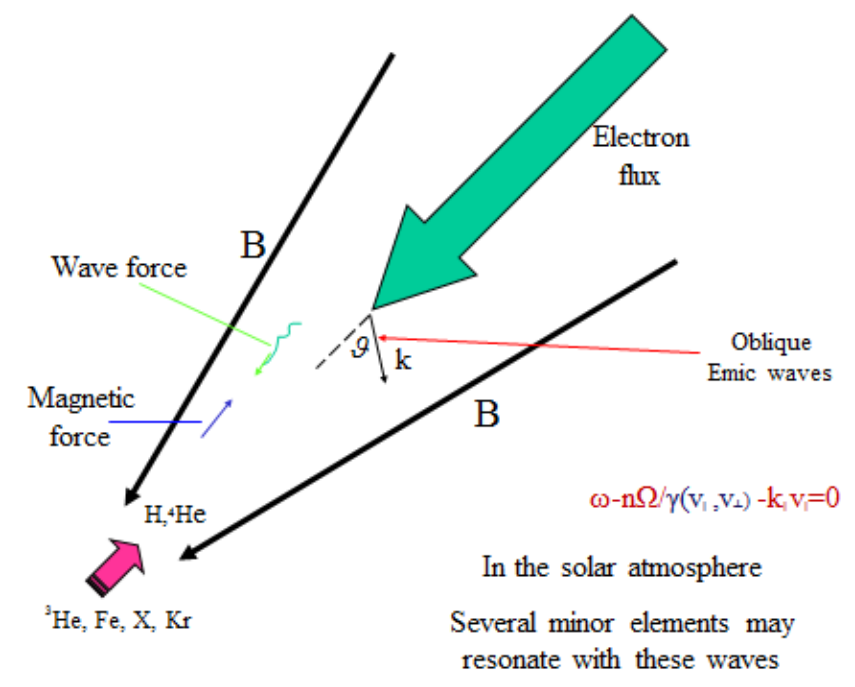

Fig. 2. Sketch of the acceleration process. Oblique waves, supported by $\mathrm{H}$ and ${ }^{4} \mathrm{He}$, interact resonantly with minority ions: ${ }^{3} \mathrm{He}$ and selected heavy ions; when the wave force balances the magnetic mirror force, the ion is efficiently energized. Correlation between ${ }^{3} \mathrm{He}$ and heavy ions (Fe) may occur only for powerful flares, when the wave amplitude becomes intense, to compensate and balance the mirror force.

acceleration of ${ }^{3} \mathrm{He}$ and ${ }^{4} \mathrm{He}$ by waves propagating parallel to the magnetic field (Petrosian and Liu, 2004), and firehose instability due to electron temperature anisotropy (Paesold et al., 2003). However, only the model of Roth and Temerin (1997) relates explicit terrestrial wave observations to processes at a similar solar configuration.

The acceleration model is based on the interaction between solar ions moving upward along the inhomogeneous magnetic field with a downward propagating oblique electromagnetic cyclotron waves (Fig. 2). In the presence of two or more ion species, this oblique wave propagates in a frequency range below the hydrogen gyrofrequency and above the twoion hybrid frequency. When the Doppler-shifted wave with frequency $\omega$ and parallel wavenumber $k_{\|}$passes through the gyrofrequency $\Omega$ of ${ }^{3} \mathrm{He}^{++}$or higher $(n>1)$ gyroharmonic of heavier ions (mainly $\mathrm{Fe}$ ), these ions are resonantly accelerated through $\omega-k_{\|} v_{\|}=n \Omega / \gamma$. In the resonance region, the propagating waves decelerate the energized upgoing ion in the parallel direction, balancing the effects of the mirror force and increasing its residence time in the interaction region, thereby enhancing the perpendicular ion heating; its gyroradius increases proportionally to $J_{n}(y) / y\left[J_{n}\left(y=k_{\perp} \rho\right)\right.$ Bessel function], and finally is ejected when the mirror force overwhelms the wave force. The $n>1$ interaction for heavier ions requires more intense waves, since the effective wave force is then much smaller for small Bessel function arguments to keep the ion in the resonance region (higher order Bessel function).
The observed charge states of the heavy elements can be used in assessing the coronal thermodynamic state and the flaring process. The temperature determines the distribution of the various charge states for each element and isotope. Inclusion of all the processes that add or subtract electrons in the atomic levels determines the ion charge equilibrium configurations. Figure 3 shows the abundances distribution of coronal ion charge states as a function of their gyrofrequencies (normalized to $\mathrm{H}$ ) at a given temperature of $6 \mathrm{MK}$. For compactness, coronal ${ }^{3} \mathrm{He}$ abundance is depicted at half of its gyrofrequency (marked on the plot). Increasing the coronal temperature shifts the distribution of the elements/isotopes to higher charge states (gyrofrequencies), indicating that the hotter flaring corona hosts significantly higher charge states than those observed in the solar wind. If one assumes that the same waves that energize ${ }^{3} \mathrm{He}$ ions also affect $\mathrm{Fe}$ ions, one would expect that the charge states of the energized Fe should be around 16-22 (half of the ${ }^{3} \mathrm{He}$ gyrofrequency). Simultaneous observations of energetic ${ }^{3} \mathrm{He}$ and $\mathrm{Fe}$ indicate more intense flares, since lower amplitude waves, although very efficient for ${ }^{3} \mathrm{He}$, have negligible efficiency in energizing Fe (higher order effect). Since at $>8 \mathrm{MK}$ the large number of ${ }^{16-22} \mathrm{Fe}$ charge states with gyrofrequencies around half of ${ }^{3} \mathrm{He}$ should allow a very large enhancement of $\mathrm{Fe} / \mathrm{O}$, while the observed values give a factor of 310 , the most favorable flare temperatures, as compared with the observed ion enhancements, are 4-6 MK. Similarly, for given observed charge states of highly stripped lighter elements, one would expect an enhanced isotopic ratio of the heavier isotope (smaller gyrofrequency), resulting in an enhanced ratio of ${ }^{22} \mathrm{Ne} /{ }^{20} \mathrm{Ne}$ and ${ }^{13} \mathrm{C} /{ }^{12} \mathrm{C}$, which may be related to the observations in planetary nebulae.

\section{Abundance anomalies in planetary nebulae}

The initial abundance of light elements is determined through the big bang nucleosynthesis (BBN), while measurements of the spatial distribution of elemental abundances provide key constraints for our understanding of galactic chemical evolution. This evolution is crucial in imposing bounds on the main BBN parameter: the baryon-to-photon ratio $\eta_{\mathrm{b}}$. The ${ }^{4} \mathrm{He}$ recombination lines in metal-poor galaxies (Peimert and Torres-Peimbert, 1974), resonance lines of ${ }^{7} \mathrm{Li}$ in metal-poor halo stars - resulting in the "Spite plateau" (Spite and Spite, 1982), Lyman series absorption lines of $D$ in metal-poor halo QSO (Tytler et al., 1996), as well as ${ }^{3} \mathrm{He}^{+}$hyperfine singletto-triplet transition (Rood et al., 1979; Bania et al., 2010) at $8.665 \mathrm{GHz}(3.46 \mathrm{~cm})$ in $\mathrm{H} \mathrm{II}$ regions allow one to estimate the $\eta_{\mathrm{b}}$ bounds, i.e., to constrain the baryon density of the Universe. Hence, ${ }^{3} \mathrm{He}$ can also be used as a "baryometer" (e.g., Yang et al., 1984).

The presently measured ${ }^{3} \mathrm{He}$ abundances in HII regions (Rood et al., 1995; Bania et al., 2010), in the proto-solar samples of gas-rich meteorites (Geiss, 1993; Galli et al., 1995), in 


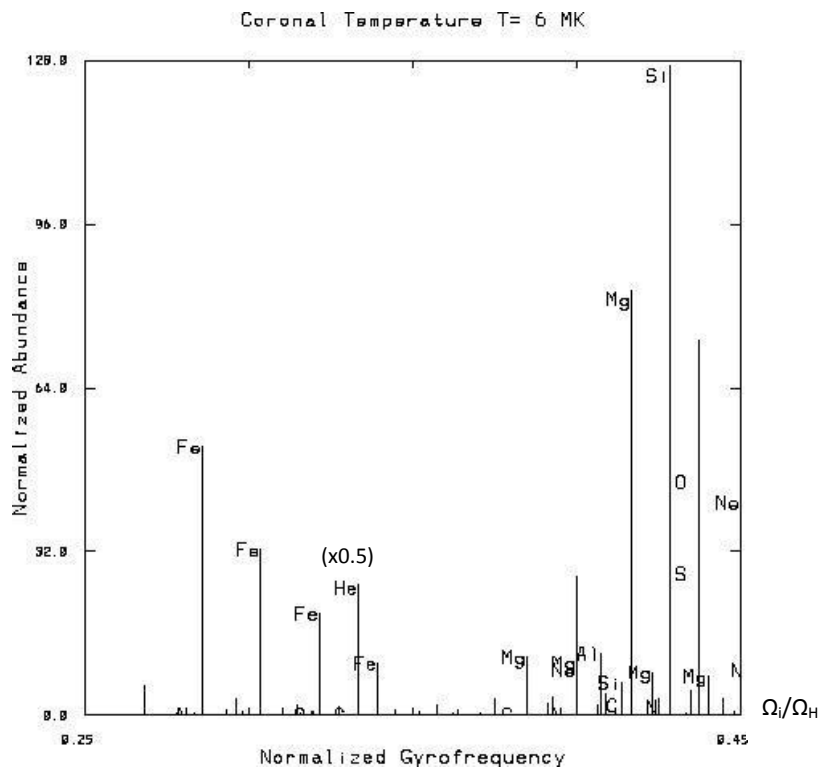

Fig. 3. Distribution of coronal elements as a function of ion gyrofrequency $\Omega_{i}$ (normalized to $\mathrm{H}$ ) at a temperature of $6 \mathrm{MK}$; He denotes the isotope ${ }^{3} \mathrm{He}$ at 0.5 of its gyrofrequency. Abundances of heavier ions are spread in gyrofrequencies according to the charge states distributions at a given coronal temperature.

the local neighborhood with pick-up ions on Ulysses spacecraft (Gloecker and Geiss, 1996), in the Jovian atmosphere by Galileo probe (Mahaffy et al., 1998), and neutral ISM fluxes on the MIR satellite (Salerno et al., 2003) are similar to the BBN yield of ${ }^{3} \mathrm{He} / \mathrm{H} \sim 2.0 \times 10^{-5}$, in disagreement with the evolutionary galactic models. Ordinary stars produce ${ }^{3} \mathrm{He}$ in the p-p burning cycle (Iben, 1967), mainly in the long living low-mass stars $\left(1-3 \mathrm{M}_{\odot}\right)$. However, no gradients in ${ }^{3} \mathrm{He} / \mathrm{H}$ from galactic center as well as in time, and no metallicity $([\mathrm{O} / \mathrm{H}])$ dependence have been observed over $30 \mathrm{yr}$ in the extremely detailed measurements (Bania et al., 2010), indicating the existence of ${ }^{3} \mathrm{He}$ plateaus and constituting the "3He problem".

Solution of the "3He problem" has been suggested due to an extra nonstandard rotation-induced and thermohaline mixing in low-mass stars at the first dredge-up on a red giant branch (RGB), which suppresses substantially the production of ${ }^{3} \mathrm{He}$ by transporting and burning ${ }^{3} \mathrm{He}$ from the convective envelope down to the hydrogen burning shell (Hogan, 1995; Charbonnel, 1995; Charbonnel and Zahn, 2007). The parametric consistency with the observed He isotope abundances in $\mathrm{H} \mathrm{II} \mathrm{regions} \mathrm{requires} \mathrm{then} \mathrm{that} 90 \%$ of the $\mathrm{M}<2.5 \mathrm{M}_{\odot}$ stars undergo the thermohaline mixing (Galli et al., 1997), similarly to the statistical study of carbon isotopes in a sample of red giant stars, which will enhance ${ }^{13} \mathrm{C} /{ }^{12} \mathrm{C}$ (Charbonnel and Nascimento, 1998). Then, consistency with the galactic evolution requires that the high measured abundance ratios of ${ }^{3} \mathrm{He} / \mathrm{H} \sim 10^{-4}-10^{-3}$ in known planetary nebulae $(\mathrm{PN})$ - ionized gas ejected during the asymptotic giant branch phase of stellar evolution - must refer only to higher mass stars, while the inferred masses of these progenitor stars show $\mathrm{M}<2 \mathrm{M}_{\odot}$, i.e., satisfying the nonstandard mixing and contradicting the measured abundances (Bania et al., 2010). Hence, a new solution is required for these $\mathrm{PN}$ observations. Another twist in the PN " $3 \mathrm{He}$ problem" was suggested (Charbonnel and Zahn, 2007) as an inhibition of the previously suggested thermoline mixing due to fossil magnetic field (a relic of the initial interstellar medium) in RGB descendants of the Ap stars (peculiar A spectral shapes).

Following the heliospheric observations of ${ }^{3} \mathrm{He} /{ }^{4} \mathrm{He}$ enhancements, it is suggested here that the PN abundances above the "3e plateau" values are formed as a result of the previously described process. Similarly to terrestrial auroral and solar observations, coronal activity at the magnetized progenitor star is efficiently accelerating ${ }^{3} \mathrm{He}$ ions via resonant interaction with ion cyclotron waves (Fig. 2). It is suggested therefore that an enrichment of a subset of minority elements at PN progenitor star results in ${ }^{3} \mathrm{He} /{ }^{4} \mathrm{He}$ enhancements in the expanding glowing shell, solving the PN "astrophysical ${ }^{3} \mathrm{He}$ problem" with important implications for galactic evolution and cosmology. Therefore, in addition to the requirements from the stellar evolution to inhibit ${ }^{3} \mathrm{He}$ in low-mass stars and deplete it in $\mathrm{H}$ II regions, one may consider a planetary nebula with its very hot central star and intense magnetic activity as a source of steady enhancement of ${ }^{3} \mathrm{He}$. This results in an average higher value of ${ }^{3} \mathrm{He} /{ }^{4} \mathrm{He}$ and ${ }^{3} \mathrm{He} / \mathrm{H}$ in the surrounding medium. The PN thermal equilibrium at the known low temperature of few eV will strip the $\mathrm{He}$ isotopes to singly charges atoms, as observed by the singlet-to-triplet transition of ${ }^{3} \mathrm{He}^{+}$. Therefore, the " ${ }^{3} \mathrm{He}$ problem" may be resolved by combined rotation-induced thermohaline instability and plasma processes in magnetized stars. This scenario may also explain the observed decreased isotopic carbon ratio, ${ }^{12} \mathrm{C} /{ }^{13} \mathrm{C}$, which does not satisfy the standard stellar evolution, while the heavier isotope is susceptible to enhanced resonant heating with the ensuing enhancement.

\section{Abundance anomalies in meteorites}

Meteorites form the raw materials for planetary formation, believed to contain dust and grain particles that accreted to form primitive asteroids in the early solar system. Most of the meteorites that reach Earth are chondrites, stony matrix material that never experienced high temperatures and have not been modified due to melting or differentiation, representing the oldest solid material within the solar system. Understanding of the early solar system must address the following topics: formation of (a) chondrules, mm-sized spherical molten igneous grain droplets, found in chondrites and (b) calcium and aluminum-rich inclusions (CAIs), mm-to$\mathrm{cm}$ calcium and aluminum-rich refractory inclusions found 
in carbonaceous chondrites - both produced by flash heat from a still controversial source, then accreted onto their parent asteroids; (c) observations of fossil short-lived (halflives of $<5 \mathrm{Myr}$ ) products of radionuclides such as ${ }^{10} \mathrm{Be}$, ${ }^{26} \mathrm{Al},{ }^{41} \mathrm{Ca}$ and ${ }^{53} \mathrm{Mn}$ in meteorites at abundances inconsistent with galactic nucleosynthesis (Lee et al., 1998; Russell et al., 2001; McKeegan and Davis, 2003).

The extinct radionuclides could have been formed either due to supernovae events or by solar cosmic ray bombardments from the young Sun (Lee et al., 1998; Gounelle et al., 2001). Of particular interest are the observations of CAI inclusions with enhanced abundance of ${ }^{26} \mathrm{Mg}$, formed by the decay of the radioactive ${ }^{26} \mathrm{Al}$ (Lee et al., 1977). The galactic isotopic ratio of ${ }^{26} \mathrm{Al} /{ }^{27} \mathrm{Al}$ (radioactive/stable) is $3 \times 10^{-6}$, while the isochron dating deduced the CAI ratio of (1.5$5) \times 10^{-5} \cdot{ }^{26} \mathrm{Al}$ decays through positron emission into the first excited state of ${ }^{26} \mathrm{Mg}^{*}$, then to its ground state, emitting $1.809 \mathrm{MeV}$ photons with a lifetime of $1.1 \times 10^{6} \mathrm{yr}$. This is one of the strongest emissions from the galactic center, indicating that presently measured ${ }^{26} \mathrm{Al}$ is created in supernovae at a rate of 3 solar masses/Myr (Prantzos and Diehl, 1996). Nucleosynthesis events cannot produce enough ${ }^{26} \mathrm{Al}$ (Cameron et al., 1995), while the bombardment of pre-solar grains by solar cosmic rays $\left(\mathrm{H},{ }^{4} \mathrm{He}\right)$ that gives consistent yield of the radioactive ${ }^{41} \mathrm{Ca}$ and ${ }^{53} \mathrm{Mn}$ results in too low a yield of ${ }^{26} \mathrm{Al}$, hence another nuclear process is required, which may be due to bombardment of the young Sun nebula by energetic flarerelated ${ }^{3} \mathrm{He}$ ions.

The young solar-like stars emit copious amount of x-rays indicating intense magnetic activity (e.g., powerful aurora), hence it is conjectured that in the early epoch the young Sun underwent frequent impulsive flares and the heliosphere was filled with a significant amount of energetic $\mathrm{MeV}^{3} \mathrm{He}$, as is observed during present active periods. The ${ }^{26} \mathrm{Al}$ isotopes were enhanced before solidification through the ${ }^{24} \mathrm{Mg}\left({ }^{3} \mathrm{He}\right.$, $\mathrm{p})^{26} \mathrm{Al},{ }^{28} \mathrm{Si}\left({ }^{3} \mathrm{He}, \alpha \mathrm{p}\right){ }^{26} \mathrm{Al},{ }^{25} \mathrm{Mg}\left({ }^{3} \mathrm{He}, \mathrm{pn}\right){ }^{26} \mathrm{Al}$, and ${ }^{27} \mathrm{Al}$ $\left({ }^{3} \mathrm{He}, \alpha\right)^{26} \mathrm{Al}$ reactions (Lee et al., 1998) by the flare-related energetic ${ }^{3} \mathrm{He}$ ions. Hence, acceleration of rare isotopes in the early solar system contributes to selective formation of radioactive elements in the early Sun, which are observed as fossil elements in meteorites.

\section{Summary}

The unique configuration of the terrestrial aurora and the detailed in situ measurements of waves and particles allow us to extrapolate these observations to solar corona and to other magnetized configurations at the birth and death of small-tomedium mass stellar systems. The enhanced abundances of coronal isotopes and charge states of heavy elements may be due to their selective interaction with waves that are correlated to electron fluxes at active corona, similarly to active auroral observations, while the anomalous abundances (a) of some radioactive daughter fossil elements in meteorites and (b) of He and heavier isotopes in planetary nebulae can be related to similar processes (1) during chondrite formation before asteroid solidification and (2) at late life stages of a small mass progenitor star that ejected its content into the stellar nebula, respectively.

Acknowledgements. This research was supported in part by NASA grant NNX09AE41G-1/14.

Topical Editor M. Balikhin thanks two anonymous referees for their help in evaluating this paper.

\section{References}

Bania, T. M., Rood, R. T., and Balser, D. S.: Measurements of ${ }^{3} \mathrm{He}$ in Galactic HII regions and planetary nebulae, in: Light Elements in the Universe, IAU Symposium 268, 81-90, edited by: Charbonnel, C., Tosi, M., Primas, F., and Chiappini, C., doi:10.1017/S174392131000390X, 2010.

Cameron, A. G. W., Hoeflich, P., Myers, P. C., and Clayton, D. D.: Massive Supernovae, Orion Gamma Rays, and the Formation of the Solar System, Astrophys. J. Lett., 447, L53-57, 1995.

Charbonell, C.: A Consistent Explanation for ${ }^{12} \mathrm{C} /{ }^{13} \mathrm{C},{ }^{7} \mathrm{Li}$ and ${ }^{3} \mathrm{He}$ Anomalies in Red Giant Stars, Astrophys. J. Lett., 453, L41, doi:10.1086/309744, 1995.

Charbonnel, C. and Do Nascimento Jr., J. D.: How many low-mass stars do destroy ${ }^{3} \mathrm{He}$ ?, Astron. Astrophys., 336, 915-919, 1998.

Charbonnel, C. and Zahn, J.-P.: Inhibition of thermohaline mixing by a magnetic field in Ap star descendants: implications for the Galactic evolution of ${ }^{3} \mathrm{He}$, Astron. Astrophys., 476, L29-L32, 2007.

Fisk, L. A.: He-3-rich flares - A possible explanation, Astrophys. J., 224, 1048-1055, 1978.

Galli, D., Palla, F., Ferrini, F., and Penco, U.: Galactic evolution of D and He-3, Astrophys. J., 443, 536-550, 1995.

Galli, D., Stanghellini, L., Tosi, M., and Palla, F.: He in Planetary Nebulae: A Challenge to Stellar Evolution Models, Astrophys. J., 477, p. 218, 1997.

Geiss, J.: Primordial abundances of hydrogen and helium isotopes, in: Origin and Evolution of the Elements, edited by: Prantzos, N., Vangioni-Flam, E., and Casse, M., Cambridge University Press, 89 pp., 1993.

Gloeckler, G. and Geiss, J.: Abundance of ${ }^{3} \mathrm{He}$ in the local interstellar cloud Nature, Nature, 381, 210-212, 1996.

Gounelle, M., Shu, F. H., Shang, H., Glassgold, A. E., Rehm, K. E., and Lee, T.: Extinct Radioactivities and Protosolar Cosmic Rays: Self-Shielding and Light Elements, Astrophys. J., 548, 10511070, 2001.

Ho, G. C., Roelof, E. C., Mason, G. M., Gold, R. E., Krimigis, S. M., and Dwyer, J. R.: Heavy ions in ${ }^{3} \mathrm{He}$ enhanced solar energetic particle events, ACE 2000 Symposium, AIP Conference Proceedings, 528, 99-102, 2000.

Hogan, C. J.: Giant branch mixing and the ultimate fate of primordial deuterium in the Galaxy, Astrophys. J. Lett., 441, L17-L20, 1995.

Hsieh, K. C. and Simpson, J. A.: Galactic ${ }^{3} \mathrm{He}$ above $10 \mathrm{MeV}$ Per Nucleon and the Solar Contributions of Hydrogen and Helium, Astrophys. J., 162, p. L197, 1970.

Iben Jr., I.: Stellar Evolution. VII. The Evolution of a $2.25 \mathrm{M}_{\text {sun }}$ Star from the Main Sequence to the Helium-Burning Phase, Astrophys. J., 147, p. 650, 1967. 
Lee, T., Papanastassiou, D. A., and Wasserburg, G. J.: Aluminum26 in the early solar system - Fossil or fuel, J. Geophys. Res., 211, L107-L110, 1976.

Lee, T., Shu, F. H., Shang, H., Glassgold, A. E., and Rehm, K. E.: Protostellar Cosmic Rays and Extinct Radioactivities in Meteorites, Astrophys. J., 506, 898-912, 1998.

Mahaffy, P. R., Donahue, T. M., Atreya, S. K., Owen, T. C., and Niemann, H. B.: Space Sci. Rev., 84, 251-263, 1998.

Mason, G. M.: The heavy-ion compositional signature in He-3-rich solar particle events, Astrophys. J., 303, 849-860, 1986.

Mason, G. M., Dwyer, J. R., and Mazur, J. E.: New Properties of ${ }^{3}$ He-rich Solar Flares Deduced from Low-Energy Particle Spectra, Astrophys. J. Lett., 545, L157-L160, 2000.

McFadden, J. P., Carlson, C. W., Ergun, R. E., Chaston, C. C., Mozer, F. S., Temerin, M., Klumpar, D. M., Shelley, E. G., Peterson, W. K., Moebius, E., Kistler, L., Elphic, R., Strangeway, R., Cattell, C., and Pfaff, R.: Geophys. Res. Lett., 25, 2045-2048, 1998.

McKeegan, K. D. and Davis, A. M.: Early Solar System Chronology Treatise on Geochemistry, in: Meteorites, Planets and Comets, edited by: Davis, A. M., Holland, H. D., and Turekian, T. T., Vol. 1, Treatise on Geochemistry, Oxford: Elsevier-Pergamon, 431 pp., 2003.

Miller, J. A. and Vinas, A. F.: Ion acceleration and abundance enhancements by electron beam instabilities in impulsive solar flares, Astrophys. J., 412, 386-400, 1993.

Peimert, M. and Torres-Peimbert, S.: Chemical composition of $\mathrm{H}$ II regions in the Large Magellanic Cloud and its cosmological implications, Astrophys. J., 193, 327-333, 1974.

Prantzos, N. and Diehl, D.: Radioactive 26Al in the galaxy: observations versus theory, Physics Reports, 267, 1-69, 1996.

Paesold, G., Kallenbach, R., and Benz, A. O.: Acceleration and Enrichment of ${ }^{3} \mathrm{He}$ in Impulsive Solar Flares by Electron Firehose Waves, Astrophys. J., 582, 495-505, 2003.
Petrosian, V. and Liu, S.: Stochastic Acceleration of Electrons and Protons. I. Acceleration by Parallel-Propagating Waves, Astrophys. J., 610, 550-571, 2004.

Reames, D. V., Meyer, J. P., and von Rosevinge, T. T.: Energeticparticle abundances in impulsive solar flare events Astrophysical Journal Supplement Series (ISSN 0067-0049), Astrophys. J. Supp., 90, 649-666, 1994.

Rood, R. T., Wilson, T. L., and Steigman, G.: The probable detection of interstellar He-3/+/ and its significance, Astrophys. J., 227, L97-L101, 1979.

Roth, I. and Temerin, M.: Enrichment of ${ }^{3} \mathrm{He}$ and Heavy Ions in Impulsive Solar Flares, Astrophys. J., 477, p. 940, 1997.

Russell, S., Gounelle, M., and Hutchison, R.: Origin of short-lived radionuclides, Philos. T. Roy. Soc. A, 359, p. 1991, 2001.

Salerno, E., Bühler, F., Bochsler, P., Busemann, H., Bassi, M. L., Zastenker, G. N., Agafonov, Yu. N., and Eismont, N. A.: Measurement of ${ }^{3} \mathrm{He} /{ }^{4} \mathrm{He}$ in the Local Interstellar Medium: the Collisa Experiment on Mir, Astrophys. J., 585, 840-849, 2003.

Spite, F. and Spite, M.: Abundance of lithium in unevolved halo stars and old disk stars - Interpretation and consequences, Astron. Astrophys., 115, 357-366, 1982.

Temerin, M. and Lysak, R. L.: Electromagnetic ion cyclotron mode (ELF) waves generated by auroral electron precipitation, J. Geophys. Res., 89, 2849-2859, 1984.

Temerin, M. and Roth, I.: The production of He-3 and heavy ion enrichment in He-3-rich flares by electromagnetic hydrogen cyclotron waves, Astrophys. J. Lett., 391, L105-L108, 1992.

Tytler, D., Fan, X. M., and Burles, S.: Cosmological baryon density derived from the deuterium abundance at redshift $z=3.57$, Nature, 381, 207-209, 1996.

Yang, J., Turner, M. S., Steigman, G., Schramm, D. N., and Olive, K. A.: Primordial nucleosynthesis - A critical comparison of theory and observation, Astrophys. J., 281, 493-511, 1984. 